\title{
LA ESPECIALIZACIÓN DE LOS PROGRAMAS EN LA UNIVERSIDAD DE NARIÑO: UN ESTUDIO DE REORGANIZACIÓN ACADÉMICO-ADMINISTRATIVA
}

\section{THE SPECIALIZATION OF THE PROGRAMS IN THE UNIVERSITY OF NARIÑO: A STUDY OF ACADEMIC AND ADMINISTRATIVE REORGANIZATION}

\section{A ESPECIALIZAÇÃO DOS PROGRAMAS NA UNIVERSIDADE DE NARIÑO: UM ESTUDO DE REORGANIZAÇÃO ACADÉMICA E ADMINISTRATIVA}

\section{Por: Edinson Ortiz Benavides}

Magíster en Economía Universidad de Los Andes. Especialista en Finanzas y Economista de la Universidad de Nariño. Profesor Asistente programa de Economía de la Universidad de Nariño. E-mail: edinsoneconomia@gmail.com

\section{RESUMEN}

El argumento central de esta investigación es que la Universidad de Nariño demanda mucha energía, tiempo y recursos en su función misional de docencia, tornándola ineficiente en flexibilidad, interdisciplinariedad e integralidad de los currículos al interior de sus programas, medidos en términos de Autoferta (AO), Coeficiente de Integración (CI), y Motricidad Dependencia (MD) respectivamente. A este respecto, la implementación de la propuesta de Especialización de los programas mejoraría de manera considerable estos indicadores y generaría un ahorro de aproximadamente $\$ 2.000$ millones de pesos cada semestre, los cuales podrían utilizarse en calidad de la docencia, investigación e interacción social.

Palabras claves: Especialización, Flexibilidad, Interdisciplinariedad, Eficiencia.

JEL: A10, I21, D61.

\section{ABSTRACT}

The central argument of this research is that the University of Nariño demand much energy, time and resources in its missionary function of teaching, mak- 
ing it inefficient in flexibility, interdisciplinarity and comprehensiveness of the curricula to the interior of its programmes, measured in terms of Autoferta (AO), coefficient of integration $(\mathrm{Cl})$, and motor unit (MD) respectively. In this regard, the implementation of the proposal of specialization of the programs would improve significantly these indicators and would generate savings of approximately 2,000 million pesos every six months, which could be used in quality of teaching, research and social interaction.

Key words: Specialization, flexibility, interdisciplinarity, efficiency.

JEL: A10, 121, D61.

\section{VISÃO GERAL}

O argumento central desta pesquisa é que a Universidade de Nariño exigem muita energia, tempo e recursos em sua função missionária do ensino, tornando-se ineficiente em flexibilidade, a interdisciplinaridade e a abrangência dos currículos para o interior dos seus programas, medido em termos de Autoferta $(\mathrm{AO})$, coeficiente de integração $(\mathrm{Cl})$ e a unidade do motor $(\mathrm{MD})$, respectivamente. Neste contexto, a implementação da proposta de especialização dos programas melhoraria significativamente estes indicadores e geraria economia de aproximadamente 2000 milhões de pesos a cada seis meses, que poderia ser usado em qualidade de ensino, pesquisa e interação social.

Palavras-chave: especialização e interdisciplinaridade, flexibilidade, eficiência.

JEL: A10, I21, D61. 


\section{INTRODUCCIÓN}

El artículo pone de manifiesto la manera cómo se viene adelantando la función de docencia en la Universidad de Nariño, como elemento central de sus procesos misionales. Igualmente, se pretende dar a conocer los beneficios que se tendrían en la formación integral de los estudiantes al darse una mayor especialización de los programas en aquellas asignaturas de su perfil, una exhaustiva revisión de sus currículos, y un redireccionamiento en la estrategia de formación.

Al configurarse la Universidad como una gran matriz, se evidenciarían las interrelaciones de oferta y demanda de asignaturas entre programas, se eliminarían a su menor expresión los intereses no académicos en la programación de los semestres y los decentes encontrarían espacios para la discusión de contenidos, metodologías y proyección de sus asignaturas.

Los currículos de cada programa deben revisarse y estructurarse coherentemente, correspondiendo a una lógica social del quehacer de la especialidad, encontrándole sentido a cada una de sus asignaturas y la interrelación entre ellas. Este ejercicio mejoraría considerablemente los indicadores de flexibilidad, interdisciplinariedad e integralidad actuales.

Para cerrar el círculo de una función de docencia con calidad y eficiencia, la Universidad de Nariño deberá cuanto antes, entrar de manera decidida en el aprovechamiento de las Tecnologías de Información y Comunicación (TIC) convirtiendo sus actuales aulas de clases en verdaderos Ambientes Virtuales de Aprendizaje (AVA), acompañado de las nuevas estrategias de formación por competencias.

El documento se encuentra estructurado en cuatro capítulos; el primero, aborda en detalle el desenvolvimiento de la función de docencia en la Universidad de Nariño. El capítulo segundo revisa los elementos teóricos sobre los cuales descansan las principales temáticas de estudio. El capítulo tercero analiza los conceptos descritos en el capítulo anterior a la luz de la información estadística disponible. Finalmente el capítulo cuarto desarrolla toda la propuesta de Especialización de los Programas en la Universidad de Nariño mostrando todas sus ventajas y beneficios.

\section{EL CONTEXTO: LA FUNCIÓN DE DOCENCIA EN LA UNIVERSIDAD DE NARIÑO}

En su Misión, "La Universidad de Nariño desde su autonomía y concepción democrática y en convivencia responsable con la región sur de Colombia, forma seres humanos, ciudadanos y profesionales en las diferentes áreas del saber y del conocimiento con fundamentos éticos y espíritu crítico, para el desarrollo alternativo en el acontecimiento mundo". Así mismo en su Proyecto Educativo Institucional (PEI) entiende la Academia como la relación entre docencia, Investigación e Interacción Social y que constituye la esencia de su quehacer universitario en respuesta a las necesidades del mundo contemporáneo. En particular, "asume la docencia como la integración de la transmisión, la apropiación, la discusión, la construcción y la producción del conocimiento, como el lugar que promueve las actitudes y las capacidades del individuo para la investigación” (Udenar, 2013). Sin embargo, en la práctica, no se dilucidan con claridad las estrategias y acciones que le permitan 
alcanzar tan nobles propósitos, pues la más elemental de sus funciones misionales, la docencia, presenta ciertas deficiencias, ocasionadas entre otras razones por la no especialización de sus programas.

En la actualidad, los 37 programas de pregrado de la Universidad de Nariño, organizados en 29 Departamentos y 11 Facultades, son prácticamente independientes. Es decir, cada programa se ofrece así mismo un porcentaje cercano o superior al $\mathbf{8 0} \%$ de las asignaturas que conforman su respectivo Plan de Estudios, generando con ello Proliferación de asignaturas de similar contenido, Dificultades en la programación académica, Alta rotación de docentes, Baja interdisciplinariedad entre programas y movilidad de estudiantes e, Ineficiencia Administrativa, a saber:

\section{a) Proliferación de Asignaturas de Similar Contenido}

En su libro La riqueza de las naciones, Adam Smith empieza con un famoso pasaje sobre la especialización del trabajo en la producción de alfileres. Smith, al describir el trabajo en una fábrica de alfileres, asentó: Un hombre tira del alambre, otro lo endereza, un tercero le saca punta y otro lo aplasta en un extremo para ponerle la cabeza. Ocho hombres trabajando de esta manera fabricaban 48.000 alfileres en un día. Sin embargo, como explica Smith, si todos hubieran trabajado por separado, en forma independiente cada uno habría producido, en el mejor de los casos 20 alfileres al día. Como observó Smith, la gran ventaja de la división del trabajo es que, al descomponer el trabajo total en operaciones pequeñas, simples y separadas, en las que los diferentes trabajadores se pueden especializar, la productividad total se multiplica en forma geométrica (monografías, 2007:1).

Estos argumentos ampliamente demostrados y aceptados universalmente, parecen no tener asiento en la función de docencia de la Universidad de Nariño. Si se mira desde una perspectiva objetiva, las personas se matriculan a un programa académico específico, a cursar un número determinado de asignaturas, que al ser aprobadas según los requerimientos de la universidad, se hacen acreedores al respectivo título profesional. Ahora bien, las Direcciones de Departamentos se interesan particularmente en ofrecerles a sus estudiantes ese $100 \%$ de asignaturas a motu proprio, olvidándose por completo de los demás programas y en ocasiones, de la calidad de las mismas.

Es muy común observar que asignaturas de un perfil económico las decidan Departamentos como Agronomía, Administración, Comercio, Sociología, Geografía, Derecho, Ingeniería de Sistemas, etc. También es común observar que asignaturas de perfil administrativo las decidan Departamentos como Economía, Comercio, Ingeniería Agroindustrial, etc. En adición, asignaturas de perfil Biológico las deciden Departamentos como Agroforestal, Agronomía, Licenciatura Con Énfasis en Ciencias Naturales. Es decir, cada departamento -se reitera- busca apresuradamente ofrecerse todos sus requerimientos de asignaturas sin contar con los otros programas. El único caso, que parece actuar coherente, y más bien, por la dificultad y especificidad de las asignaturas, es el del programa de matemáticas, que provee casi todas las asignaturas de su perfil a todos los programas de la universidad.

En diversas situaciones, las direcciones de Departamentos con anuencias de sus Comités Curriculares alteran el nombre de ciertas asignaturas para acuñarlas por la 
fuerza a su perfil. Lo común es utilizar los adjetivos: "con énfasis en”, "aplicada a”, "dirigido a", entre otros. Por ejemplo, economía colombiana como asignatura, debe ser la misma en cualquier departamento que se demande. Lo mismo debe acontecer con Introducción a la Economía, Microeconomía, Formulación de Proyectos, Biología I, Metodología de la Investigación, Matemáticas Financieras (Ingeniería Económica), Teoría y Política Monetarias, Planeación Económica, Matemáticas I, Principios de Administración, entre muchísimas otras.

\section{b) Dificultades en la Programación Académica}

Lo expuesto anteriormente, es causa principal de que semestre tras semestre las Direcciones de Departamentos se encuentren en una carrera sin precedentes al final e inicio de éstos, intentando "cuadrar" la carga académica. Es común que entradas algunas semanas del semestre no se tengan cubiertas algunas asignaturas de su responsabilidad, que si bien es cierto en la sede central - Pasto - la frecuencia es menor, en sus Sedes o Extensiones se ratifica este hecho.

La programación académica como componente fundamental de la función de docencia de la universidad, demanda en las condiciones actuales mucha energía, tiempo y dedicación, restándole posibilidades de atender decididamente las funciones complementarias de investigación e interacción social, e insertarse efectivamente al panorama regional, nacional e internacional.

\section{c) Alta Rotación de Docentes}

Lo expuesto hasta ahora, genera en las condiciones actuales una alta rotación de docentes, que se contratan esporádicamente por una o dos asignaturas, y que coincidencialmente el fraccionamiento de asignaturas entre docentes aumenta en épocas pre-electorales, o lo que es lo mismo, la relación asignaturas/docentes disminuye. Esto ocurre precisamente porque los programas no se han especializado en las asignaturas de su perfil, y por otro lado, existen muchos "nombres y apelativos" de asignaturas de similar contenido curricular, dando mayor margen de "maniobra" a las direcciones de departamento o decanaturas y a la vez, mayor complejidad a la programación académica.

\section{d) Baja Interdisciplinariedad entre programas y movilidad de estudiantes}

La Universidad de Nariño en su Proyecto Educativo asume como características inherentes a sus programas académicos: la contextualización, la pertinencia, la interdisciplinariedad y la transdisciplinariedad como atributos de la flexibilidad curricular. Igualmente, sus programas se proyectarán hacia el reconocimiento y la movilidad nacional e internacional de estudiantes y profesores (Udenar, 2013).

En este sentido, "el tema de la interdisciplinariedad surge en la universidad como respuesta a los múltiples cuestionamientos sobre la forma común de sumatoria de asignaturas como se organizan los currículos, y los consecuentes métodos de enseñanza e investigación. Son frecuentes las críticas a la atomización del saber, a la organización universitaria en unidades académicas aisladas y cerradas en sí mismas, a la organización de la enseñanza en tiempos y horarios establecidos rígidamente 
para cada disciplina en el plan de estudios y a las metodologías tradicionales de enseñanza que llevan a la pasividad, repetición acrítica de contenidos y dogmatismo. La interdisciplinariedad se fundamenta ontológicamente en una concepción de la realidad como totalidad dinámica, cuyos procesos se dan por la interacción de sus elementos. La separación metodológica que se hace de los elementos de la realidad es para favorecer su estudio y análisis, en una primera etapa del proceso de conocimiento. Pero no se puede perder la dimensión de totalidad, la realización de la integración y síntesis. Epistemológicamente, implica una concepción del proceso de producción de conocimiento de la realidad, que se sectoriza en objetos propios de diversos estudios (pluralidad de ciencias), pero que en otro momento requiere la articulación de las ciencias, respetando su relativa autonomía, pues en última instancia, los problemas que la realidad plantea son asuntos complejos que una ciencia no puede resolver por sí misma, y que implican el aporte e interrelación con otras ciencias que abordan la misma realidad, desde diferentes y particulares perspectivas”. (Panqueva, 2007:1).

En consecuencia, la Universidad de Nariño se aparta de estos principios al estar muchos de sus programas aislados y sin posibilidades de interrelación en actividades curriculares para estudiantes de semestres o programas distintos. Nuevamente esta situación es generada por la no especialización de los programas, manifestándose en una escasez relativa y subutilización de salones para algunos programas, pugnas entre ellos, arduo trabajo de los comités curriculares aprobando solicitudes de estudiantes para cursar asignaturas en otros programas, aislamiento total entre docentes y estudiantes de una misma facultad o de facultades afines, etc. De la misma manera este aislamiento no permite una mayor movilidad interna de estudiantes ni la realización de trabajos de grados ni proyectos de investigación conjuntos entre diferentes programas, negándose la posibilidad de un aporte más significativo a la ciencia y la sociedad.

\section{e) Ineficiencia Administrativa}

Entendida la Eficiencia como la óptima utilización de los recursos disponibles para la obtención de resultados deseados, la no especialización de los programas en la Universidad de Nariño genera efectos contrarios en su función de docencia. Es común escuchar en las Direcciones de Departamentos al final de cada semestre, con miras a la planeación del semestre entrante, la frase "no hay salones", teniendo en muchas ocasiones que enviar a estudiantes cuya sede principal es Torobajo, a recibir algunas clases en las instalaciones de la Vipri. Para dar un ejemplo concreto, estudiantes de economía deben recibir, en ocasiones, clases en el bloque 2, bloque tecnológico, edificio de ingeniería y Vipri, a veces de manera simultánea en un mismo semestre académico. Pero también es frecuente observar salones donde solo reciben clases un número reducido de estudiantes, asignaturas idénticas que también están recibiendo un grupo reducido de estudiantes en otro salón pero de otro programa, o cuya asignatura presenta una adjetivación. Esta situación genera ineficiencia en la utilización tanto de los recursos físicos como humanos de la institución, pues si se organizan los cursos de la manera descrita en la especialización de los programas, dichos recursos adquieren mayor eficiencia en su utilización. 
Edinson Ortiz Benavides

La especialización de los programas en la Universidad de Nariño:

Un estudio de reorganización académico-administrativa

\section{MARCO DE REFERENCIA}

La presente investigación se enmarca en los conceptos de interdisciplinariedad, flexibilidad curricular e integralidad, componentes fundamentales del currículo, que en el ejercicio de la docencia suele confundirse con los conceptos de disciplina o profesión.

Según Senge, una disciplina es un cuerpo teórico y técnico que se debe estudiar y dominar para llevarlo a la práctica, que permite adquirir ciertas aptitudes y competencias, lo cual supone un compromiso constante con el aprendizaje, es decir, pasar la vida dominando disciplinas (Senge, 1996: 20). Una profesión en cambio, se entiende como una práctica socialmente legítima debido a la "posesión de conocimientos científicos, humanísticos o artísticos especializados, adquiridos por medio de un estudio formal acreditado de una manera, y cuyo ejercicio público se hace a cambio de una remuneración” (Vázquez, 1982: 1).

El currículo por su parte, se concibe como una propuesta construida colectivamente para propiciar experiencias de aprendizaje, que en la mayor parte de los casos se plantea explícita y formalmente, pero de cualquier manera también refleja intencionalidades generalmente ocultas, subyacentes a las prácticas y a los planteamientos formales (Furlan, 1981: 110-119).

La estructura curricular, por tanto, se entiende como un conjunto de componentes organizadores en relación con los fines de la educación, contenidos, experiencias formativas, recursos y valoraciones, a partir de las cuales se definen los planes de estudio. La estructura curricular de un programa es, en cierta forma, "la columna vertebral de los procesos formativos, pues de ella depende la orientación, la selección, la organización y la distribución de los conocimientos y de las prácticas que contribuyan con la formación profesional”.

Al confundir estos conceptos, también se confunden los campos curriculares, los campos disciplinarios y los campos profesionales en que cada uno se desenvuelve, siendo que tienen diferentes objetos: la acreditación, el conocimiento y el empleo, respectivamente. Cabe sin embargo esperar todo tipo de interacciones no solamente entre las disciplinas, sino también entre currículos y entre profesiones. Cualquiera de estos tres tipos de interacción constituye interdisciplinariedad, flexibilidad e integralidad.

Definida la interdisciplinariedad como aquella que transversa las funciones sustantivas de la institución y se constituye en base para la generación de conocimiento teórico y aplicado partiendo del reconocimiento de la complejidad de la realidad en sus diversas esferas, a partir de la cooperación entre las disciplinas y profesiones, la flexibilidad curricular se concibe como la propiedad inherente al currículo, que ofrece al estudiante la posibilidad de relacionarse con el entorno natural, social, político, cultural y económico de la región y acercarse al mundo dinámico de las ciencias y la tecnología. La flexibilidad curricular permite entonces la articulación entre la academia, la administración, la sociedad y la organización de los proyectos educativos por programa, de acuerdo con los diferentes modos de producción del conocimiento y de las transformaciones culturales del medio (Udenar, 2013). En lo fáctico, la flexibilidad curricular en el plano local es aquella que permite a 
los estudiantes la definición de su inclinación profesional o estudiar conforme su ritmo intelectual o de disponibilidad de tiempo o condiciones económicas. Esto es, que el currículo de su carrera le permita una mayor movilidad universitaria y posibilidades de escoger su ritmo académico sin ataduras de muchos prerrequisitos, escoger una rama de estudio dentro de su profesión y posibilitarle inclusive una doble titulación. En el plano nacional e internacional la flexibilización curricular debería permitirles a los estudiantes la inserción exitosa en programas afines a su carrera en estos contextos.

En lo que respecta a la integralidad del currículo, "al considerar las ciencias, la filosofía, las artes y la literatura como expresiones de la dimensión humana, la Universidad de Nariño, fiel a su Visión y Misión, exalta al ser humano como sujeto histórico y creador de cultura, como centro esencial del quehacer académico. Bajo esta concepción humanística, se requiere una formación integral que aborde los fundamentos éticos, estéticos y epistémicos de las diferentes expresiones del ser humano, y que construya nuevas alternativas de vida” ((Udenar, 2013).

Una vez analizadas las características curriculares de flexibilidad, interdisciplinariedad e integralidad, éstas se cuantificarán a través de los indicadores de Autoferta (AO), Coeficiente de Integración (CI), y Motricidad Dependencia (MD) respectivamente, para así obtener una medida numérica sobre el impacto o beneficio de la propuesta de implementación de la Especialización de los programas en la Universidad de Nariño.

\section{Auto-Oferta (AO)}

Este indicador representa el porcentaje de asignaturas que un programa ofrece de manera autónoma. Es decir, el total de asignaturas ofrecidas por los docentes adscritos al programa, sobre el total de asignaturas que integran el respectivo plan de estudios:

$$
A O=\frac{D_{i, i}}{D_{i}} \times 100
$$

Dónde:

$\mathrm{AO}=$ Auto-Oferta

$\mathrm{D}_{\mathrm{i}, \mathrm{i}}=$ Total de asignaturas demandas por el programa $\mathrm{i}$, y ofrecidas por el mismo programa i.

$\mathrm{D}_{\mathrm{i}}=$ Total de asignaturas demandadas por el programa i. Es decir, total de signaturas que integran el plan de estudios, excepto las institucionales como formación humanística, lecto-escritura y herramientas informáticas.

$\mathrm{i}=1,2,3, \ldots \mathrm{n}$. Siendo $\mathrm{n}=$ número total de programas en la universidad

Este indicador se lo toma como variable proxy de la flexibilidad en el entendido que una mayor auto-oferta de los programas limita las posibilidades de los estudiantes de relacionarse con su entorno. 


\section{Coeficiente de Integración (CI)}

El Coeficiente de Integración es una medida de cuan integrado se encuentran los programas al interior de un Departamento, Facultad o Universidad. Expresa el porcentaje de asignaturas demandadas y ofrecidas mutuamente por los programas que integran la respectiva unidad académica. Es decir, el total de asignaturas ofrecidas mutuamente sobre el total de asignaturas demandadas por los respectivos programas:

$$
C I=\frac{\sum_{i, j=1}^{k} D_{i, j}}{\sum_{i=1}^{k} D_{i}} x 100 \quad \forall i \neq j
$$

Dónde:

$\mathrm{CI}=$ Coeficiente de Integración

$\sum_{i, j=1}^{k} D_{i, j}=$ Total de asignaturas demandadas por los programa i, y ofrecidas por los programas $\mathrm{j}$. indica que se excluye la auto-oferta.

$\sum_{i=1}^{k} D_{i}=$ Total de asignaturas demandadas por los programas i que integran el respectivo departamento, facultad o universidad.

$\mathrm{i}=1,2,3, \ldots \mathrm{k}$. Siendo $\mathrm{k}=$ número total de programas en el departamento, facultad o universidad.

Este indicador se lo empareja con la interdisciplinariedad debido a que expresa el grado de relación de los diferentes programas al interior de una misma unidad académica, sea departamento, facultad o universidad completa.

\section{Motricidad-Dependencia (MD)}

Este es un indicador adaptado del método estructural que analiza la interrelación entre variables en estudios prospectivos. En este caso particular, representa el peso porcentual que cada programa tiene sobre el total de asignaturas ofrecidas (motricidad) y demandadas (dependencia) en la universidad de estudio. Es decir, el total de asignaturas ofrecidas y demandadas por un programa sobre el total de asignaturas ofrecidas en la universidad, excluida la auto-oferta:

Dónde:

$$
\mathrm{MD}=\mathrm{M}+\mathrm{D} ; \quad M=\frac{O_{i, j}}{\sum_{i, j=1}^{n} O_{i, j}} x 100 ; \quad D=\frac{D_{i, j}}{\sum_{i, j=1}^{n} D_{i, j}} \times 100
$$

MD = Motricidad-Dependencia

$\mathrm{M}=$ Motricidad

$\mathrm{D}=$ Dependencia

$\mathrm{O}_{\mathrm{i}, \mathrm{j}}=$ Total de asignaturas ofrecidas por el programa i a los programas $\mathrm{j}$ 
$\sum_{i, j=1}^{n} O_{i, j}=$ Total de asignaturas ofrecidas por los programa i, y demandadas por los programas j. $\forall i \neq j$ indica que se excluye la auto-oferta.

$\mathrm{D}_{\mathrm{i}, \mathrm{j}}=$ Total de asignaturas demandadas por el programa i de los programas $\mathrm{j}$

$\sum_{i j=1}^{n} D_{i, j}=$ Total de asignaturas demandas por los programa i, y ofrecidas por los programas j. $\forall i \neq j$ indica que se excluye la auto-oferta.

$\mathrm{i}=1,2,3, \ldots \mathrm{n}$. Siendo $\mathrm{n}=$ número total de programas en la universidad

Este indicador se lo asocia con la característica de integralidad del currículo porque evidencia de alguna manera la relevancia del programa al interior de la universidad. Para esto es preciso, clasificar los programas en algunos de los cuadrantes resultantes de diagramar estos indicadores en un plano cartesiano donde sus ejes se dividen con el cálculo de la Esperanza Matemática (E) que resulta de suponer a todos los programas como independientes:

$$
E=\frac{100 \%}{n}
$$

Dónde:

$\mathrm{E}=$ Esperanza Matemática

$\mathrm{n}=$ número total de programas en la universidad.

Los datos anteriores se llevan a un plano cartesiano y se obtiene el siguiente esquema:

Figura 1. Diagrama de Motricidad - Dependencia. Método Estructural

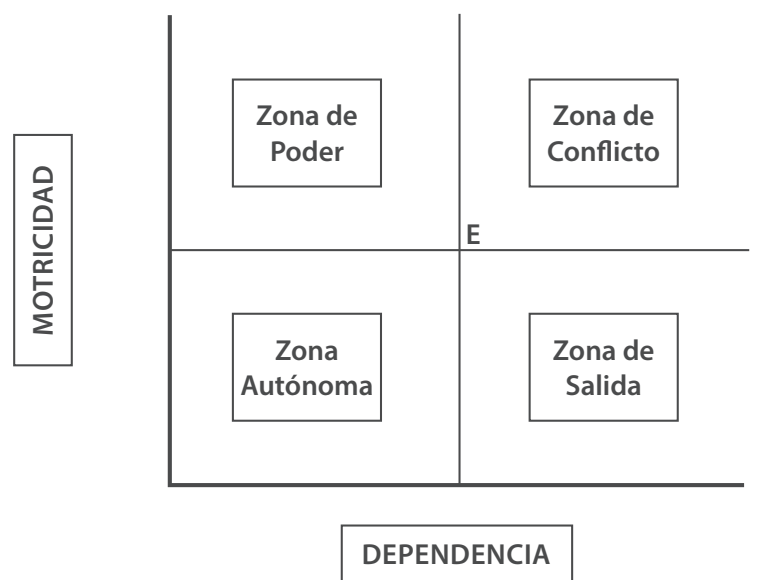

Fuente: Esta Investigación. 
Edinson Ortiz Benavides

La especialización de los programas en la Universidad de Nariño:

Un estudio de reorganización académico-administrativa

En la zona de poder.- Se ubican los programas con una alta influencia sobre los demás programas de la universidad, pero con poca influencia de ellos. Muestra los programas con una alta integralidad de su currículo.

En la zona de conflicto.- Se ubican los programas con una alta influencia sobre los demás programas de la universidad, pero también con alta influencia de ellos.

En la zona de salida.- Se ubican los programas con una baja influencia sobre los demás programas de la universidad, pero con alta influencia de ellos.

En la zona autónoma.- Se ubican los programas con una baja o nula influencia sobre los demás programas de la universidad y también con una muy baja o nula influencia de ellos. Muestra los programas prácticamente autónomos con un contacto autista con el contexto universitario y que a nuestro juicio tienen muy baja integralidad de su currículo.

\section{ANÁLISIS DE DATOS}

Los 37 programas de pregrado de la Universidad de Nariño, 29 Departamentos y 11 Facultades con sus 1.836 asignaturas se pueden organizar en una gran matriz de Oferta/Demanda donde se entrecrucen las asignaturas (en número) que cada programa se ofrece así mismo y las asignaturas que ofrece a los otros programas de toda la universidad. Igual para el caso de la demanda. Esta matriz permite obtener los indicadores de Auto-oferta, Coeficiente de Integración y de MotricidadDependencia descritos con anterioridad, obteniéndose los siguientes resultados:

\section{Auto-Oferta (AO)}

Aplicando la fórmula de $\mathrm{AO}$ a la matriz descrita anteriormente se obtiene que a nivel de programas, el promedio de la Auto-oferta es de 83,1\%, es decir, en este porcentaje los programas de la Universidad de Nariño se ofrecen a sí mismo sus asignaturas dejando un margen muy reducido para el contacto de sus estudiantes con otros contextos, es decir, baja flexibilidad (1-0,831 = 16,9\%). El 51\% de los programas se encuentran por encima de este promedio. Sobresalen los programas de la Facultad de Artes, de Educación y Psicología que se ofrecen así mismos el 100\% de las asignaturas de su Plan de Estudios. En contraste, Contaduría Pública y Licenciatura en humanidades se ofrecen así mismos el 55,6\% y el 54,4\% de las asignaturas de su pensum respectivamente.

Si el análisis se efectúa por facultades, se encuentra que las facultades de Artes, Ciencias de la Salud, Ciencias Humanas, Ciencias Pecuarias, Derecho y Educación se ubican por encima del promedio que es de 82,4\%. Las Facultades de Ciencias Económicas y Administrativas, Ciencias Agrícolas, Ciencias Exactas y Naturales, Ingeniería e Ingeniería Agroindustrial se ubican por debajo de este promedio, dando un mayor margen de flexibilidad a sus estudiantes por encima del 17,6\% de las asignaturas de sus respectivos planes de estudio. 


\section{Coeficiente de Integración (CI)}

Aplicando la fórmula de CI a la matriz descrita, La Facultad de Ciencias Exactas y Naturales y de Ciencias Económicas y Administrativas son quienes mayor interdisciplinariedad presentan con indicadores de coeficiente de integración de 15,6\% y 14,7\%. Las facultades de Artes, Ciencias de la Salud, Derecho, Educación y Agroindustria son quienes presentan menor interdisciplinariedad, pues sus coeficientes de integración se ubican por debajo del 1\%.

\section{Motricidad-Dependencia (MD)}

Respecto a este indicador los programas de la Universidad de Nariño presentan un promedio de $5,4 \%\left(2{ }^{\star} \mathrm{E}\right)$, es decir en este porcentaje ofrecen y demandan asignaturas a los otros programas de la Universidad. Sobresale el programa de Licenciatura en Matemáticas que cuenta con una motricidad-dependencia de 35,1\%, seguido de Física y Química con un 12,6\% programas adscritos a la misma facultad. Les sigue el programa de Economía con un 9,9\% perteneciente a la Facultad de Ciencias Económicas y Administrativas. De la misma manera sobresalen todos los programas de la Facultad de Artes, Licenciatura en Lengua Castellana y Literatura, con indicadores de Motricidad- Dependencia iguales a cero, es decir, con ninguna integración e interdisciplinariedad con los demás programas de la Universidad.

Esta información se aprecia con mayor claridad en la figura 2, donde se establece la zona a la que cada programa se adscribe dadas sus características de MD, ratificando lo anterior en el sentido de la relevancia de los programas en la Universidad de Nariño. Se aprecia que sobre la Zona de Poder se ubican los programas de Lic. en Matemáticas (25), Lic. en Inglés Francés (20), Psicología (21), Física (24) y Derecho (31). En la Zona de Conflicto se ubican los programas de Química (27), Biología (23), Economía (11) e Ing. en Sistemas (35). El resto de programas se ubican en las Zonas de Salida y Autónoma, como aquellos que presentan menor influencia (Motricidad) sobre los demás programas de la Universidad de Nariño.

A nivel de facultades en consecuencia de lo anterior, el promedio de MotricidadDependencia se ubica en 18,2\%, sobresaliendo la Facultad de Ciencias Exactas y Naturales con un $72,2 \%$ de $\mathrm{MD}$ que vincula los programas de mayor $\mathrm{MD}$ en la Universidad, le siguen en importancia la Facultad de Ciencias Económicas y Administrativas (Facea) y la Facultad de Ciencias Humanas con indicadores de MD $38,7 \%$ y $30,5 \%$ respectivamente. Las demás facultades se encuentran por debajo del promedio, pero se destacan las Facultades de Artes, Ciencias de la Salud, y de Educación que cuentan con una interdisciplinariedad de cero o muy cercana a cero.

\section{LA ESPECIALIZACIÓN DE LOS PROGRAMAS}

Conforme lo anterior, se observa que los programas de la Universidad de Nariño en la actualidad cuentan con características de flexibilidad, interdisciplinariedad e integralidad deficientes, pero susceptibles de mejorar. En este sentido la propuesta de Especialización de los Programas definida como el escenario donde los programas se especializan en ofrecer aquellas asignaturas de su perfil específico, plantea: 


\section{Redefinición de Oferentes de Asignaturas}

La Especialización de los Programas plantea el reconocimiento de una especie de "propiedad intelectual" sobre cada asignatura. Por ejemplo debe definirse quien posee la autoridad profesional y moral sobre asignaturas como Ingeniería Económica (Matemáticas Financieras), Formulación y Evaluación de Proyectos, Metodología de la Investigación, Economía Agraria o Ambiental, Pedagogías, entre otras. De esta manera una signatura será ofrecida únicamente por aquel programa que tenga el perfil idóneo y la experticia tanto para sí mismo como para todos los demás programas de la Universidad, acabando de paso con la solicitud de los mal llamados "servicios" que cuando un Director de Departamento no tiene "carga" para sus docentes, sobretodo de cátedra o servicios prestados, simplemente no los solicita, por el contrario, se los ofrece autónomamente, afectando con ello la calidad de dicha asignatura y por ende la formación de sus estudiantes. Este reconocimiento obligaría a que algunos programas cedan ciertas asignaturas a sus verdaderos especialistas, y acojan aquellas de las cuales ellos son idóneos.

Figura 2. Zonas de Motricidad - Dependencia Actual de Programas

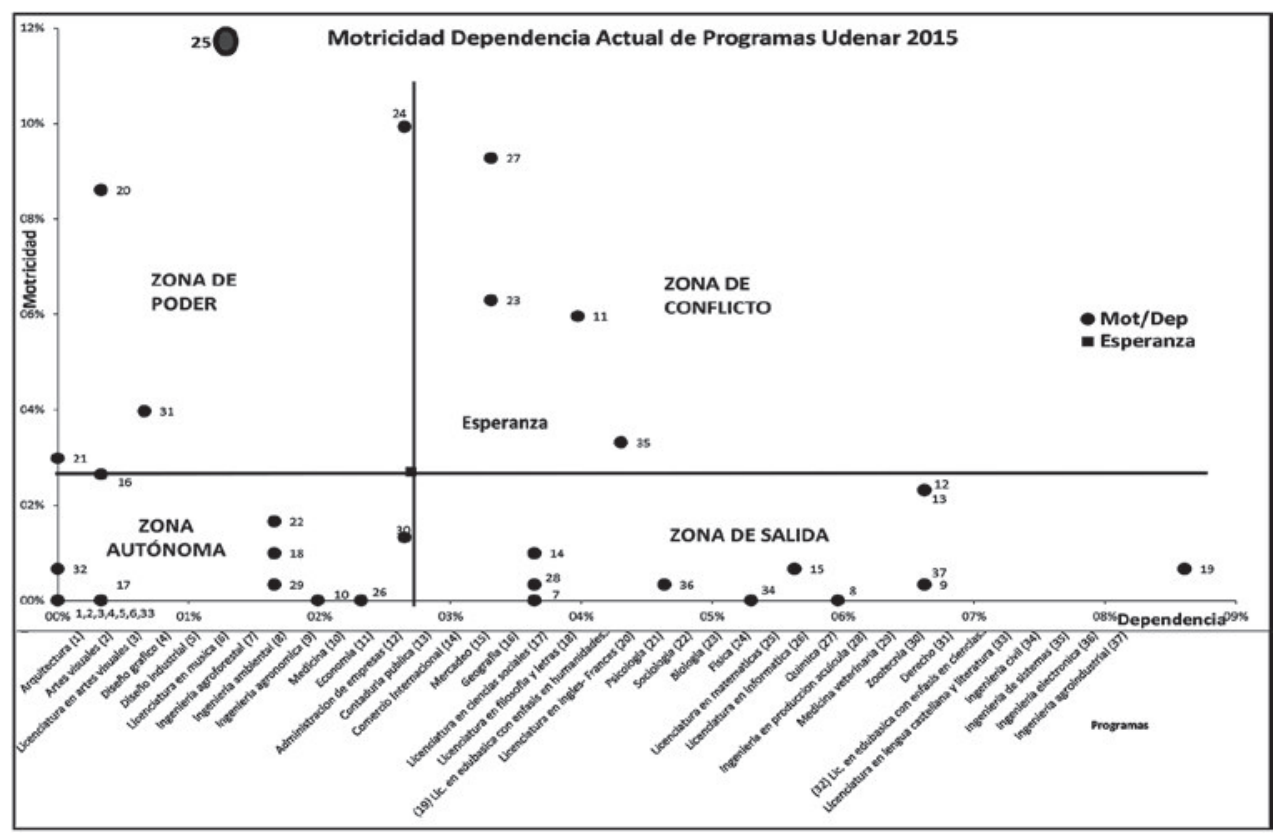

Fuente: Esta Investigación. Cálculos propios.

"La universidad es una sola", y si al final lo que ofrece son un determinado número de asignaturas, entonces debería interesarse por la especialización de las mismas, para mejorar su calidad, y así darle la eficiencia y acabado a su función docencia, para avanzar con éxito y sin contratiempos a las funciones investigativa y de interacción social, que completan la triada de su Misión - Visión. 
En este nivel de análisis, la Universidad debe poner sobre el tapete las 1.836 asignaturas que actualmente ofrece y objetivamente conforme su estructura temática, enmarcarla dentro del perfil de uno u otro programa, pero no en varios a la vez. Esto es, especializar los programas, llegando a explotar con mayor intensidad sus capacidades y se ofrecerían mejor calidad a sí mismos y a los otros programas que demanden sus asignaturas, incrementando con ello, el nivel de formación profesional en toda la Universidad.

Es oportuno considerar por parte de las Direcciones de Departamento, que si bien representan uno o más programas de pregrado, su mayor responsabilidad es con la universidad en general, es decir, con el ofrecimiento de las asignaturas de su perfil para todos los programas que las demanden, pues el pregrado que representan no se nutre únicamente con asignaturas de su especialidad, requiere por el contrario de las demás ciencias que le aportan una formación integral y coherente con la realidad y necesidades contextuales a sus estudiantes. Las Direcciones de Departamento llegarían entonces a ocupar un espacio de mayor relevancia y trascendencia en la universidad, que al tradicionalmente confinado.

Figura 3. Auto-oferta Propuesta de Programas

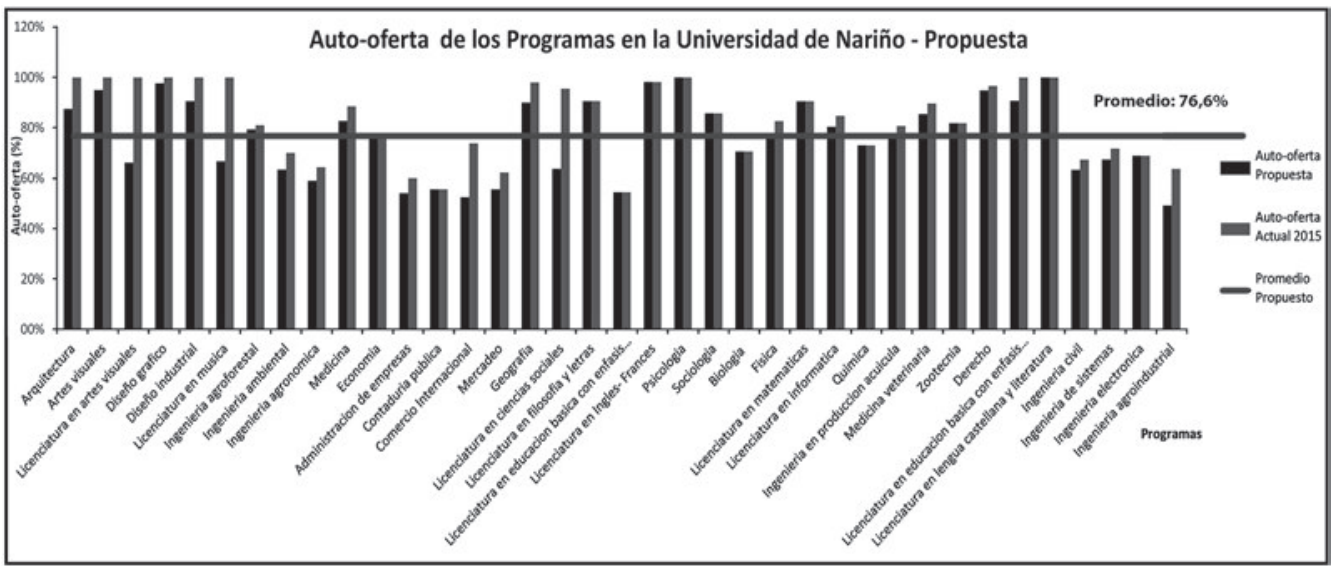

Fuente: Esta Investigación.

Cuando se implementan estos cambios, los indicadores de flexibilidad (AO), interdisciplinariedad (CI) e integralidad (MD) se incrementan de manera importante. A nivel de programas la flexibilidad se incrementa en 6,5 puntos porcentuales, al pasar el promedio de AO de 83,1\% a 76,6\% (Ver Figura 3).

Como se observa el total de programas de la Universidad reducen su Autooferta, es decir aumentan su flexibilidad. Sobresalen Artes visuales, Licenciatura en Música, Comercio Internacional y Licenciatura en Ciencias Sociales que en promedio aumentan su flexibilidad en 30 puntos porcentuales. 
A nivel de facultades, el panorama es similar, la flexibilidad aumentaría 6,1 puntos porcentuales al pasar el promedio de AO de 82,4\% a 76,3\%. (Ver Figura 4).

Figura 4. Auto-oferta Propuesta de Facultades

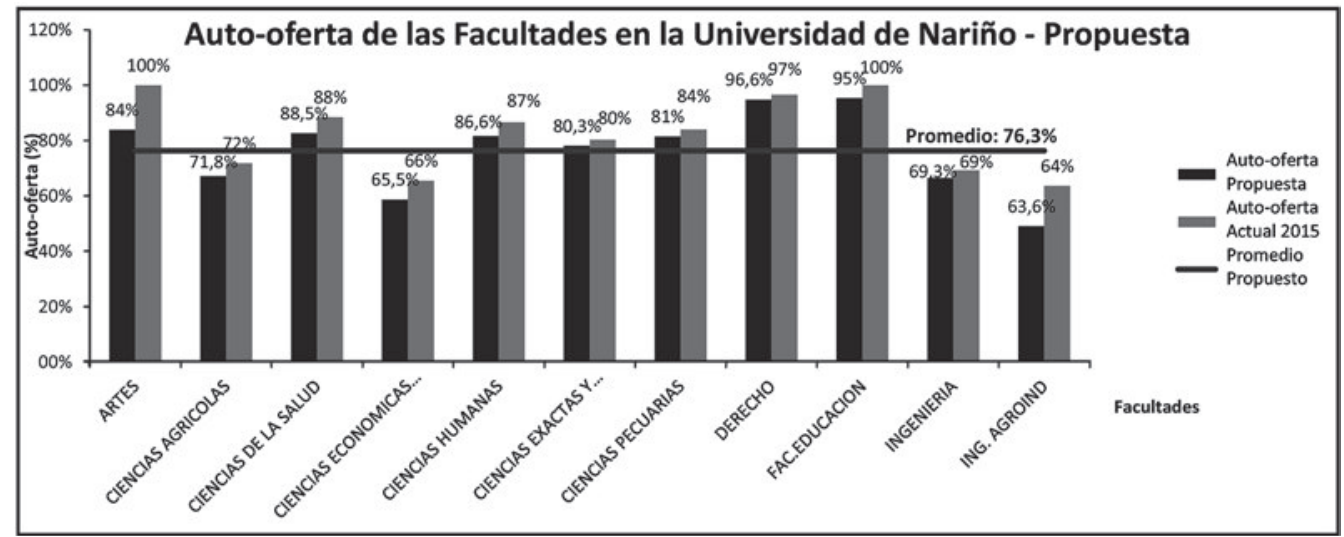

Fuente: Esta Investigación.

Las facultades que más aumentarían su flexibilidad curricular serían en su orden, Artes con 16,1 puntos porcentuales (pp), Agroindustrial con 14,5 pp, Facea 6,8 pp y Ciencias Humanas con 5 pp.

En relación con la interdisciplinariedad, en promedio las facultades solo aumentan un punto porcentual, pero en toda la universidad el indicador de Coeficiente de Integración aumenta 6,8 puntos porcentuales al pasar el promedio de CI de $16,4 \%$ a $23,2 \%$ (Ver Figura 5).

Figura 5. Coeficiente de Integración Propuesto de Facultades

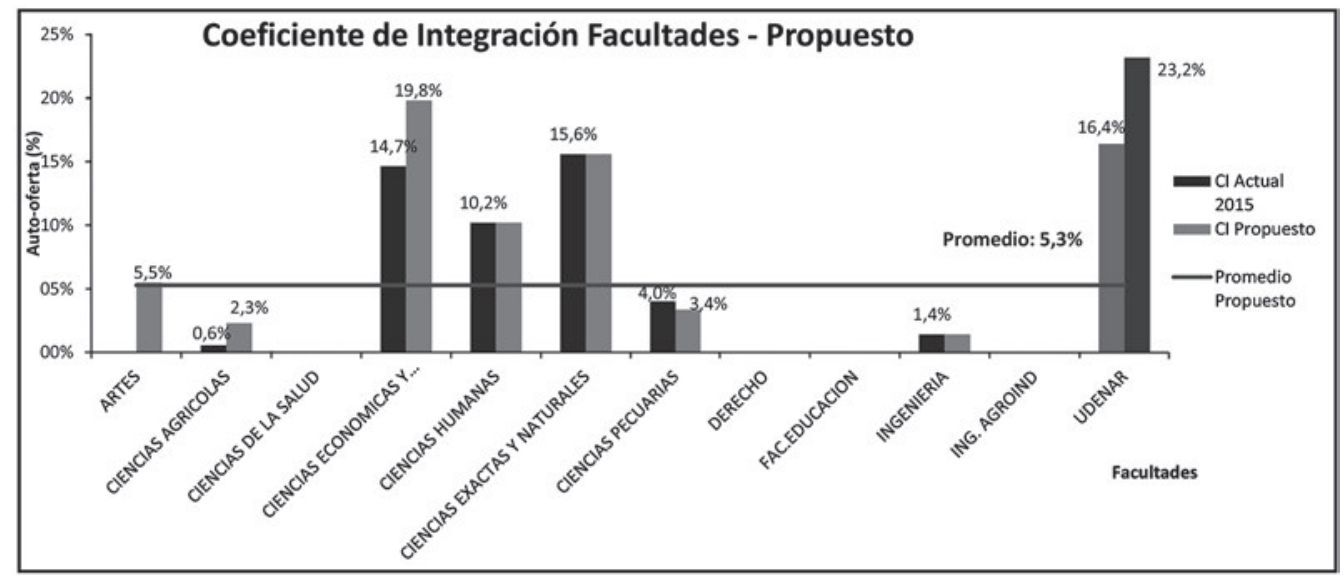

Fuente: Esta Investigación. 
Las facultades que más aumentarían su interdisciplinariedad serían la Facea, Ciencias Agrícolas, y Artes. Muchas facultades todavía mantienen un CI igual a cero sobretodo porque tienen un único programa de pregrado o porque los programas que tienen en la facultad tienen poca relación, obligando esto a la reubicación de las mismas.

Por su parte, la integralidad que por razones matemáticas no presenta variación en los promedios a nivel de programas y facultades pero si lo hace en la desviación estándar. A nivel de programas la desviación estándar del indicador de MD se reduce 1,8 puntos porcentuales al pasar de $6.2 \%$ a $4,4 \%$ aplicando la propuesta de especialización de programas, reflejando una menor dispersión de los datos, o mayor cohesión de los programas. Se observaría un aumento importante de la integralidad en los programas de la facultad de Artes y Educación, pero se reduce en los programas de Ciencias Exactas e Ingenierías (Ver Figura 6).

Figura 6. Motricidad - Dependencia Propuesta de Programas

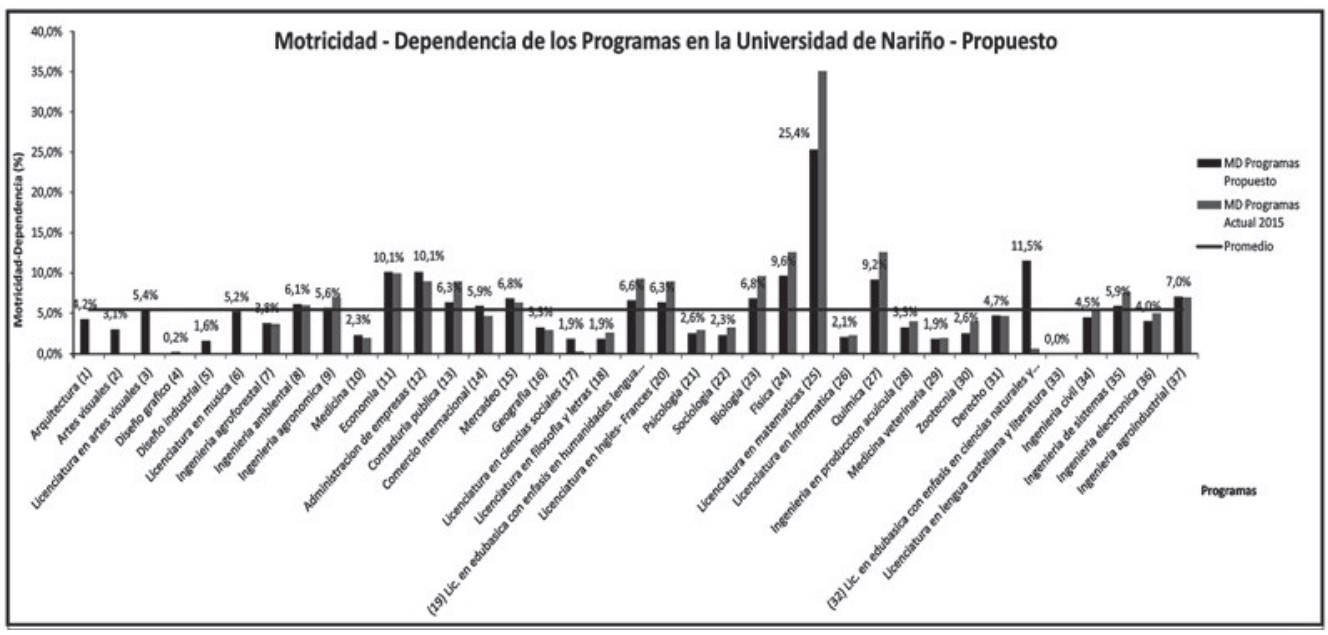

Fuente: Esta Investigación.

A nivel de facultades la desviación estándar del indicador de MD se reduce 6,2 puntos porcentuales al pasar de $21,8 \%$ a $15,6 \%$ aplicando la propuesta de especialización de programas. Facultades que ganan integralidad serían Artes, Educación y Facea, las otras verían sacrificada un poco este indicador (Ver Figura 7).

\section{Revisión Planes de Estudios}

La Especialización de los Programas plantea entonces Eliminar las duplicidades de asignaturas con adjetivos y apelativos en la Universidad. Al eliminarse la solicitud actual de servicios, los programas deberán ajustar sus Planes de Estudios y establecer las asignaturas que serán ofrecidas por sí mismos y aquellas que serán demandadas a otros programas de la Universidad, constituyéndose así las Áreas de 
Formación Disciplinar y de Formación Complementaria respectivamente, que junto con las Áreas de Formación Humanística y de Formación Básica (Lecto-escritura, Herramientas Informáticas, Inglés) constituyen los Planes de Estudios. Cada área de formación a su vez, contará con sub-áreas de conformidad con las directrices institucionales y de cada Departamento.

Figura 7. Motricidad - Dependencia Propuesta de Facultades

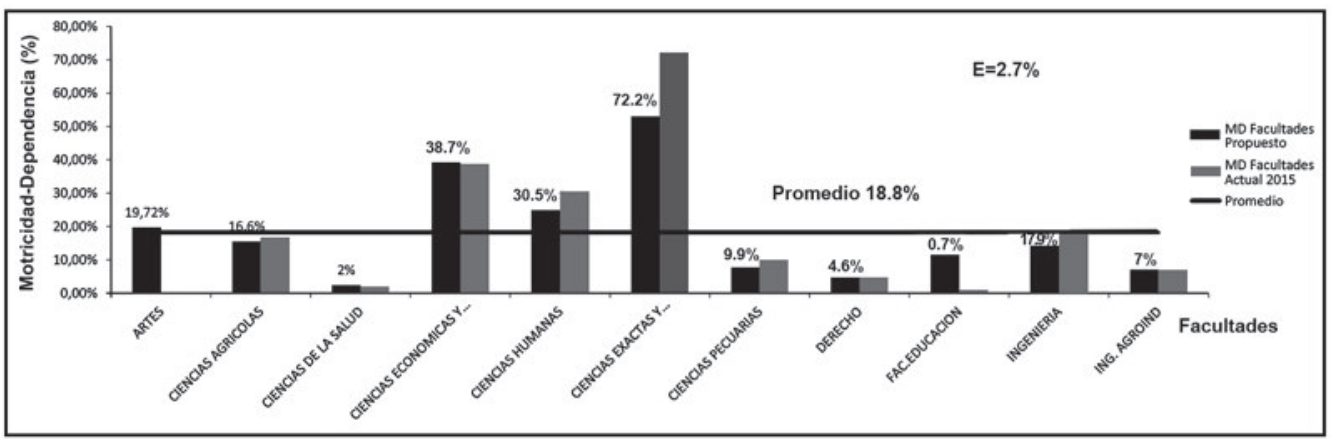

Fuente: Esta Investigación.

\section{Reorganización de Facultades}

La propuesta de Especialización de los Programas también permite obtener una propuesta más objetiva para la reorganización de facultades, pues debido a que devuelve la autoridad a los programas sobre las asignaturas de su perfil, esto permite saber qué programas se encuentran más interrelacionados con otros y por ende pueden crear una facultad más eficiente, mejor estructurada y más cohesionada.

La propuesta de la reorganización de facultades plantea la siguiente estructura (Ver Figura 8).

Esta propuesta contempla pasar de 11 a 8 facultades, fusionándose las facultades de Agroindustria, Ciencias Pecuarias y Ciencias Agrícolas en la Facultad de Ciencias Agropecuarias. Así mismo las facultades de Ingeniería y algunos programas de la Facultad de Artes se fusionan en la Facultad de Ingeniería y Diseño. La Facultad de Derecho y algunos programas de la Facultad de Ciencias Humanas se fusionan en la nueva Facultad de Ciencias Políticas y Sociales. Otros programas de la Facultad de Ciencias Humanas se reorganizan en la Facultad de Educación y en la de Ciencias de la Salud. Esta propuesta de reorganización en general plantea una mejoría en los indicadores de Flexibilidad Curricular e Interdisciplinariedad tanto a nivel de Facultades como de toda la Universidad (Ver Figura 9).

Como se observa la Flexibilidad Curricular aumenta tanto en las nuevas facultades como la Universidad en general al aumentar en 4,6 y 6,5 puntos porcentuales los indicadores de Flexibilidad (1-AO) respectivos. La Interdisciplinariedad también se incrementa bajo este nuevo escenario; el coeficiente de integración en 
las facultades se incrementa 2,5 puntos porcentuales, y en toda la universidad lo hace en $6,8 \mathrm{pp}$.

Figura 8. Propuesta de Reorganización de Facultades

\begin{tabular}{|c|c|c|c|c|c|c|c|c|c|c|c|c|c|}
\hline FACULTAD & \multicolumn{3}{|c|}{ ARTES } & \multicolumn{7}{|c|}{ CIENCIAS AGROPECUARIAS } & \multicolumn{2}{|c|}{ CIENCIAS DE LA SALUD } & \\
\hline DEPARTAMENTO & \multicolumn{2}{|c|}{ Artes Visuales } & \multirow{2}{*}{ 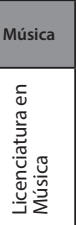 } & \multicolumn{2}{|c|}{ Rec. Nat. y Sist. Agrof. } & \multirow{2}{*}{ 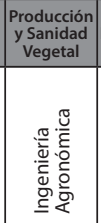 } & \multirow{2}{*}{ 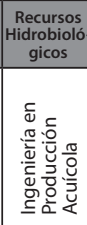 } & \multirow{2}{*}{ 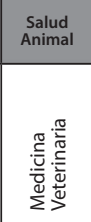 } & \multirow{2}{*}{ 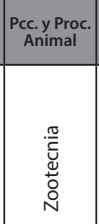 } & \multirow{2}{*}{ 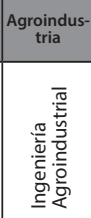 } & \multirow{2}{*}{$\begin{array}{c}\begin{array}{c}\text { Salud } \\
\text { Humana }\end{array} \\
\\
\\
. \frac{\pi}{5} \\
. \frac{0}{0} \\
\sum^{\frac{0}{\Sigma}}\end{array}$} & \multirow{2}{*}{\begin{tabular}{|c|} 
Psicología \\
\\
$\frac{0}{\frac{0}{5}}$ \\
$\frac{0}{0}$ \\
$\frac{0}{n}$ \\
0
\end{tabular}} & \\
\hline 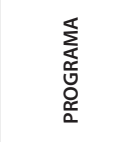 & 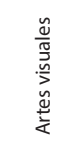 & 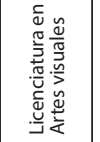 & & 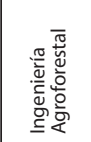 & 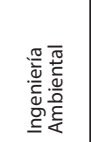 & & & & & & & & \\
\hline FACULTAD & \multicolumn{5}{|c|}{ CIENCIAS ECONÓMICAS Y ADMINISTRATIVAS } & \multicolumn{4}{|c|}{ CIENCIAS POLITICAS Y SOCIALES } & \multicolumn{4}{|c|}{ CIENCIAS NATURALES } \\
\hline DEPARTAMENTO & Economía & $\begin{array}{r}\text { Admón. de } \mathrm{E} \\
\text { Finan }\end{array}$ & $\begin{array}{l}\text { mpresas y } \\
\text { zas }\end{array}$ & $\begin{array}{r}\text { Comercio In } \\
\text { y Mer }\end{array}$ & $\begin{array}{l}\text { ternacional } \\
\text { cadeo }\end{array}$ & Geografía & $\begin{array}{l}\text { Ciencias } \\
\text { Sociales }\end{array}$ & $\begin{array}{c}\text { Derecho } \\
\text { y Ciencias } \\
\text { Politicas }\end{array}$ & Sociología & Biología & Física & $\begin{array}{c}\text { Matemá- } \\
\text { ticasy } \\
\text { Estadistica }\end{array}$ & Química \\
\hline 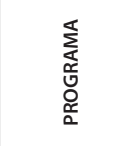 & 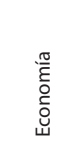 & 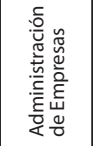 & 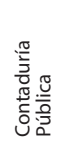 & 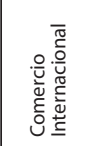 & $\begin{array}{l}0 \\
\frac{d}{0} \\
\frac{\pi}{0} \\
\frac{0}{\Sigma}\end{array}$ & 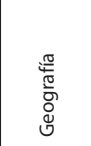 & 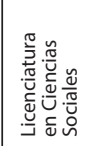 & 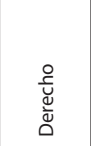 & 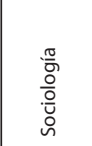 & $\frac{\frac{\pi}{5}}{\frac{0}{0}}$ & 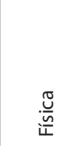 & 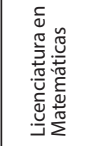 & 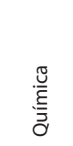 \\
\hline FACULTAD & \multicolumn{6}{|c|}{ EDUCACIÓN } & \multicolumn{6}{|c|}{ INGENIERIA Y DISEÑO } & \\
\hline DEPARTAMENTO & $\begin{array}{l}\text { Humani- } \\
\text { dades y } \\
\text { filosofia }\end{array}$ & \multicolumn{2}{|c|}{ Lingüistica e Idiomas } & Informática & \multicolumn{2}{|c|}{ Estudios Pedagógicos } & Ing. Civil & Sistemas & Electrónica & $\begin{array}{c}\text { Arquitec- } \\
\text { tura }\end{array}$ & \multicolumn{2}{|c|}{ Diseño } & \\
\hline 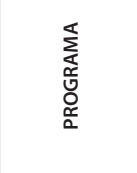 & 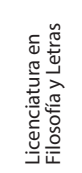 & 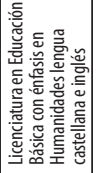 & 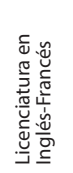 & 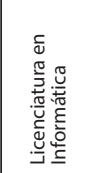 & 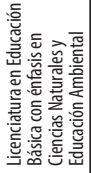 & 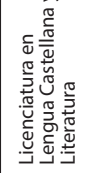 & 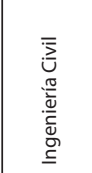 & 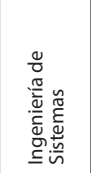 & 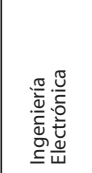 & 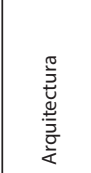 & 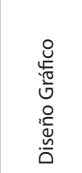 & 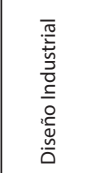 & \\
\hline
\end{tabular}

Figura 9. Propuesta de Reorganización de Facultades (NF)

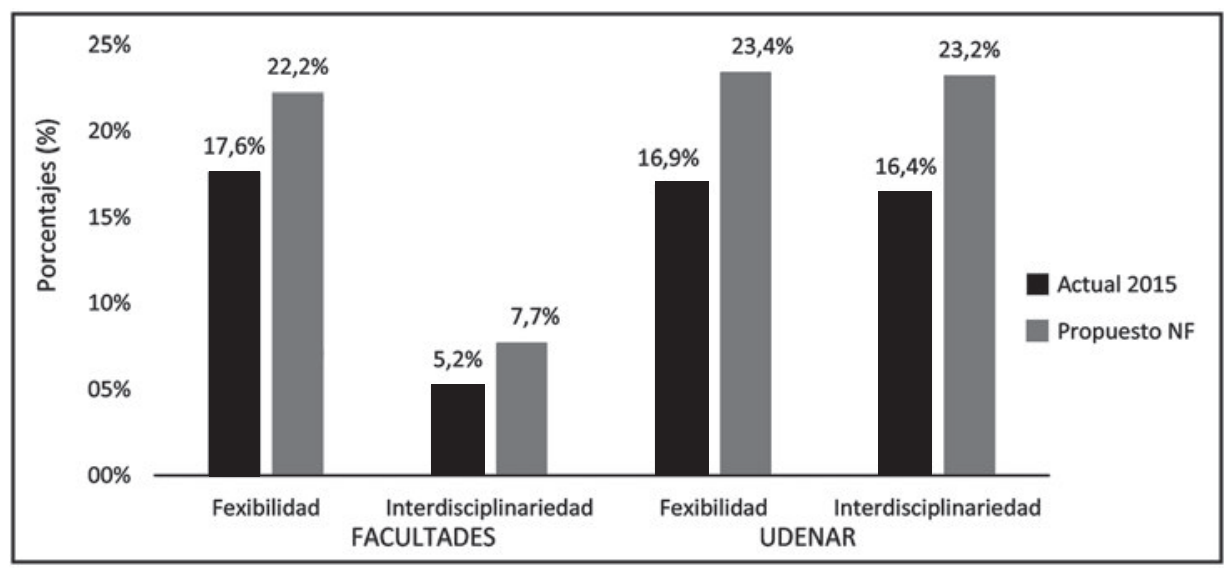

Fuente: Esta Investigación. 
Edinson Ortiz Benavides

La especialización de los programas en la Universidad de Nariño:

Un estudio de reorganización académico-administrativa

\section{Reasignación de Recursos Físicos, Humanos y Financieros}

La implementación de la Especialización de los programas de la mano con la reorganización de las facultades plantea también el reto de que tanto las instalaciones físicas, como el personal de docentes y los recursos financieros adquieran mayor eficiencia en su utilización, creando las condiciones para el cambio en la estrategia de formación.

En primera instancia se espera que cada facultad tenga un contacto mayor con sus programas y no estén tan dispersos como en la actualidad. En este sentido, al especializarse cada programa en el ofrecimiento de las asignaturas de su perfil, deberá contar con la logística suficiente para tal fin, de tal manera que los otros programas que requieran cursar dichas asignaturas lo hagan en sus dominios, propiciándose una mayor integración y convivencia universitaria, pues estudiantes de diferentes programas se darían cita en un recinto donde se ofrezca una asignatura de interés para ellos, bien sea que haga parte de su formación complementaria o una electiva como elemento de flexibilidad en su Plan de estudios. De esta manera se lograría una ocupación superior al 70\%, en salones con capacidad para 60 estudiantes, logrando mayor eficiencia en la utilización de los mismos, evitando de paso que se abran cursos idénticos en un mismo semestre en diferentes programas simultáneamente con ocupaciones inferiores en los salones. Esta eficiencia también se logra en utilización de aulas de informáticas, laboratorios, granjas y demás unidades de apoyo a la docencia.

En segunda instancia, eliminar duplicidad en asignaturas conllevaría a que se cuente con el número de docentes necesarios para su ofrecimiento, sin que existan docentes atendiendo a un número reducido de estudiantes en asignaturas que se estén ofreciendo simultáneamente en otros contextos, al menos en los semestres iniciales. Actualmente la universidad cuenta con 687 docentes laborando en 1987 cursos para 9.047 estudiantes que los toman, dando como resultado un promedio de 24,3 estudiantes por cursos, si elevamos este promedio a 40 estudiantes, tendríamos una reducción aproximada de 774 cursos correspondiente al 39,1\%, que en términos financieros representaría un ahorro aproximado de \$2.000 millones de pesos cada semestre que podrían utilizarse en calidad de la docencia, investigación e interacción social. Un ejemplo concreto es Matemáticas I, o Matemáticas generales el cual es demandado por muchos programas en un mismo semestre, la propuesta es que el departamento de matemáticas ofrezca un número de cursos que garanticen un promedio de estudiantes por curso superior a 40 estudiantes dándole a ellos la oportunidad de escogencia de docente y horario.

Respecto a la estrategia de formación, "hasta hace poco tiempo, (realidad actual para la Universidad de Nariño) los salones de clase se limitaban al pizarrón, los pupitres, los lápices, los cuadernos y libros de texto. Ahora, en las aulas de formación, hoy denominados Ambientes Virtuales de Aprendizaje (AVA), éstos elementos se alternan con modernas tecnologías, para que el alumno interactúe y colabore con su guía (antes, el profesor) y con otros compañeros, desarrolle com- 
petencias y adquiera conocimientos a través de esas herramientas o Tecnologías de la Información y la Comunicación (TIC) disponibles.

Pero, todo esto no es un asunto de moda. Es consecuencia de las nuevas realidades de la economía mundial y de los avances tecnológicos, que están empujando la transición de una economía de capital a una economía basada en el conocimiento. Es una nueva sociedad que permea todos los aspectos de la vida cotidiana de un país: lo económico, lo laboral, lo social, lo recreativo, lo educativo y lo relativo a la formación para el trabajo" (Cuestas, 2006: 8-9).

\section{Redefinición Programación Académica}

La Especialización de los programas mediante la reorganización de facultades y la mejor asignación de cursos físicos, humanos y financieros lleva también implícito un cambio en la actual forma de programar académicamente los semestres.

Actualmente existe lo que denomino una programación por oferta, esto es, que los programas ofrecen a los estudiantes un determinado conjunto de asignaturas y un salón específico para los mismos. Con la Especialización de los programas la programación será por demanda, es decir, que serán los estudiantes quienes demandan a los programas las asignaturas según sus preferencias por docentes y disponibilidad de horario. Esto reflejaría mayor flexibilidad e interdisciplinariedad de los programas y contribuiría a propiciar un ambiente más universitario.

\section{CONCLUSIONES}

En la actualidad, los 37 programas de pregrado de la Universidad de Nariño, organizados en 29 Departamentos y 11 Facultades, son prácticamente independientes. Cada programa se ofrece así mismo un porcentaje correspondiente al 83\% de las asignaturas que conforman su respectivo Plan de Estudios, generando con ello: Proliferación de asignaturas de similar contenido, Dificultades en la programación académica, Alta rotación de docentes, Baja interdisciplinariedad entre programas y movilidad de estudiantes e, Ineficiencia Administrativa.

Esta situación se genera principalmente por la "no especialización de los programas" ocasionando ineficiencias en las características de flexibilidad, interdisciplinariedad e integralidad de los currículos al interior de estos programas medidas en términos de Autoferta (AO), Coeficiente de Integración (CI), y Motricidad Dependencia (MD) respectivamente. Conforme la revisión de datos, a nivel de programas, el promedio de la Auto-oferta es de 83,1\%, el Coeficiente de Integración de 16,4\% y el indicador de motricidad-dependencia es de 5,4\%.

Conforme lo anterior, se observa que los programas de la Universidad de Nariño en la actualidad cuentan con características de flexibilidad, interdisciplinariedad e integralidad muy deficientes pero susceptibles de mejorar. En este sentido, al implementarse la propuesta de Especialización de los Programas definida como el escenario donde los programas se especializan en ofrecer aquellas asignaturas de 
su perfil específico, los indicadores de flexibilidad (AO), interdisciplinariedad (CI) e integralidad (MD) se incrementan de manera importante. A nivel de programas la flexibilidad se incrementa en 6,5 puntos porcentuales, al pasar el promedio de AO de $83,1 \%$ a 76,6\%. En relación con la interdisciplinariedad el indicador de Coeficiente de Integración aumenta 6,8 puntos porcentuales al pasar el promedio de CI de $16,4 \%$ a $23,2 \%$. Por su parte, la integralidad que por razones matemáticas no presenta variación en los promedios a nivel de programas y facultades pero si lo hace en la desviación estándar. A nivel de programas la desviación estándar del indicador de MD se reduce 1,8 puntos porcentuales al pasar de $6.2 \%$ a $4,4 \%$ aplicando la propuesta de especialización de programas, reflejando una menor dispersión de los datos, o mayor cohesión de los programas.

La propuesta de Especialización de los Programas también permite obtener una propuesta más objetiva para la reorganización de facultades, pues debido a que devuelve la autoridad a los programas sobre las asignaturas de su perfil, esto permite saber qué programas se encuentran más interrelacionados con otros y por ende pueden crear una facultad más eficiente, mejor estructurada y más cohesionada. La propuesta contempla pasar de 11 a 8 facultades.

Finalmente, la implementación de la Especialización de los programas de la mano con la reorganización de las facultades plantea también el reto de que tanto las instalaciones físicas, como el personal de docentes y los recursos financieros adquieran mayor eficiencia en su utilización, creando las condiciones para el cambio en la estrategia de formación. En concreto, si elevamos el promedio de estudiantes por cursos de 24,3 a 40 estudiantes, se tendría una reducción aproximada de 774 cursos correspondiente al 9,1\%, que en términos financieros representaría un ahorro aproximado de $\$ 2.000$ millones de pesos cada semestre, que podrían utilizarse en calidad de la docencia, investigación e interacción social. 


\section{BIBLIOGRAFÍA}

1. CUESTAS CUELLAR, Gabriel (2006): La Formación de la mano de la Tecnología. En: Formar - revista del Sena. Santa Fe de Bogotá, No. 11 de septiembre-octubre.

2. FURLAN, A. (1981): "El currícum pensado y el currículum vivido". En:V jornadas sobre problemas de enseñanza aprendizaje en el área de la salud. ENEP - Iztacala, UNAM. México.

3. Monografías (2007): La División del Trabajo de Adam Smith. En: (www.monografías.com/trabajos6/ diop/diop.shtml). San Juan de Pasto, mayo 17.

4. PANQUEVA, Tarazona (2007): Javier. La Interdisciplinariedad y el Currículo Integrado. En: www.google. com. San Juan de Pasto, Mayo 3 de 2007.

5. SENGE, Peter M. (1996): La quinta disciplina. El arte y la práctica de la organización abierta al aprendizaje. Barcelona: Granica.

6. UNIVERSIDAD DE NARIÑO-Udenar (2013): Proyecto Educativo Institucional. San Juan de Pasto. P. 10.

7. VAZQUEZ, J.Z. (1982): Historia de las profesiones en México. Colegio de México, SEP-SESIC. México. 